\title{
Optimal and game ship control algorithms for avoiding collisions at sea
}

\author{
J. Lisowski \\ Department of Ship Automation, Gdynia Maritime University, Poland
}

\begin{abstract}
This paper introduces the application of optimal and game theory methods in marine navigation. The functional scope of a standard ARPA anti-collision system ends with the simulation of the manoeuvre altering course or speed selected by the navigator. The problem of selecting such a manoeuvre is very difficult as the process of control is very complex and game making in its nature. The most adequate model of the process that has been adopted is a model of a differential game. The control goal is defined firstly, followed by a description of the base model and a presentation of approximated models. For each approximated model, an appropriate method of safe control to support the navigator decision in a collision situation has been assigned. The POSitional TRAJectory (POSTRAJ) and the RISK TRAJectory (RISKTRAJ) control algorithms have been designed. The considerations have been illustrated in examples of computer simulation algorithms to determine the safe ship trajectories in situations when passing many objects.

Keywords: differential games, positional games, matrix games, dual linear programming, safety navigation, transport engineering, safe ship operations.
\end{abstract}

\section{Differential game model of the ship control process}

The process of ship control in collision situations, when a great number of objects is encountered, often occurs under the conditions of indefiniteness and conflict, accompanied by an inaccurate co-operation of the objects within the context of the International Regulations for Preventing Collision at Sea (COLREG). The most adequate model of the process that has been adopted is a model of a differential game, in general of $j$ tracked ships as control objects $[2,8]$. 


\subsection{State equation}

The properties of the process are described by the state equation:

$$
\begin{array}{r}
\dot{x}_{i}=f_{i}\left[\left(x_{0}^{\vartheta_{0}}, x_{1}^{\vartheta_{1}}, \ldots, x_{j}^{\vartheta_{j}}, \ldots, x_{m}^{\vartheta_{m}}\right),\left(u_{0}^{v_{0}}, u_{1}^{v_{1}}, \ldots, u_{j}^{v_{j}}, \ldots, u_{m}^{v_{m}}\right), t\right] \\
i=1,2, \ldots,\left(j \cdot \vartheta_{j}+\vartheta_{0}\right), j=1,2, \ldots, m
\end{array}
$$

where

$\vec{x}_{0}^{\vartheta_{0}}(t)-\vartheta_{0}$ dimensional vector of the process state of the own ship determined in time span $t \in\left[t_{0}, t_{k}\right]$,

$\vec{x}_{j}^{\vartheta_{j}}(t)-\vartheta_{j}$ dimensional vector of the process state for the $j$-th object,

$\vec{u}_{0}^{v_{0}}(t)-v_{0}$ dimensional control vector of the own ship,

$\vec{u}_{j}^{v_{j}}(t)-v_{j}$ dimensional control vector of the $j$-th object, see fig. 1 .

Taking into consideration the equations describing the own ship's hydromechanics and equations of the own ship's movement relative to the $j$-th encountered object, the equations of the general state of the process (1) take the following form:

$$
\begin{aligned}
\dot{x}_{0}^{1}= & x_{0}^{2} \\
\dot{x}_{0}^{2}= & a_{1} x_{0}^{2} x_{0}^{3}+a_{2} x_{0}^{3}\left|x_{0}^{3}\right|+b_{1} x_{0}^{3}\left|x_{0}^{3}\right| u_{0}^{1} \\
\dot{x}_{0}^{3}= & a_{4} x_{0}^{3}\left|x_{0}^{3}\right|\left|x_{0}^{4}\right| x_{0}^{4}\left(1+x_{0}^{4}\right)+a_{5} x_{0}^{2} x_{0}^{3} x_{0}^{4}\left|x_{0}^{4}\right|+a_{0} x_{0}^{2} x_{0}^{3} x_{0}^{4}+a_{7} x_{0}^{3}\left|x_{0}^{3}\right|+ \\
& +a_{8} x_{0}^{5}\left|x_{0}^{5}\right| x_{0}^{6}+b_{2} x_{0}^{3} x_{0}^{4}\left|x_{0}^{3} u_{0}^{1}\right| \\
\dot{x}_{0}^{4}= & a_{3} x_{0}^{3} x_{0}^{4}+a_{4} x_{0}^{3} x_{0}^{4}\left|x_{0}^{4}\right|+a_{5} x_{0}^{2} x_{0}^{4}+a_{9} x_{0}^{2}+b_{2} x_{0}^{3} u_{0}^{1} \\
\dot{x}_{0}^{5}= & a_{10} x_{0}^{5}+b_{3} u_{0}^{2} \\
\dot{x}_{0}^{6}= & a_{11} x_{0}^{6}+b_{4} u_{0}^{3} \\
\dot{x}_{j}^{1}= & -x_{0}^{3}+x_{j}^{2} x_{0}^{2}+x_{j}^{3} \cos x_{j}^{3} \\
\dot{x}_{j}^{2}= & -x_{0}^{2} x_{j}^{1}+x_{j}^{3} \sin x_{j}^{3} \\
\dot{x}_{j}^{3}= & -x_{0}^{2}+b_{4+j} x_{j}^{3} u_{j}^{1} \\
\dot{x}_{j}^{4}= & a_{11+j} x_{j}^{4}\left|x_{j}^{4}\right|+b_{5+j} u_{j}^{2}
\end{aligned}
$$

The state variables are represented by the following values:

$x_{0}^{1}=\psi$ - course of the own ship,

$x_{0}^{2}=\dot{\psi}-$ angular turning speed of the own ship, 
$x_{0}^{3}=V-$ speed of the own ship,

$x_{0}^{4}=\beta$-drift angle of the own ship,

$x_{0}^{5}=n$ - rotational speed of the screw propeller of the own ship,

$x_{0}^{6}=H$ - pitch of the adjustable propeller of the own ship,

$x_{j}^{1}=D_{j}$ - distance to the $j$-th object,

$x_{j}^{2}=N_{j}$ - bearing of the $j$-th object,

$x_{j}^{3}=\psi_{j}$ - course of the $j$-th object,

$x_{j}^{4}=V_{j}$ - speed of the $j$-th object.

where $\vartheta_{0}=6, \vartheta_{j}=4$.

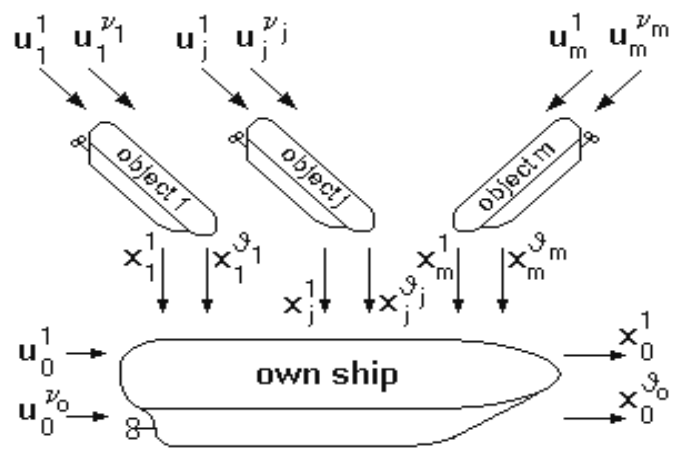

Figure 1: Block diagram of a differential game model including $j$ ships.

While the control values are represented by:

$u_{0}^{l}=\alpha_{r}$ - reference rudder angle of the own ship,

$u_{0}^{2}=n_{r}$ - reference rotational speed of the own ship's screw propeller,

$u_{0}^{3}=H_{r}$ - reference pitch of the adjustable propeller of the own ship,

$u_{j}^{1}=\psi_{j}$ - course of the $j$-th object,

$u_{j}^{2}=V_{j}$ - speed of the $j$-th object.

where $v_{0}=3, v_{j}=2$.

\subsection{Control and state constraints}

The constraints of the control and the state of the process are connected with the basic condition for the safe passing of the objects at a safe distance $D_{s}$ in compliance with COLREG Rules, generally in the following form: 


$$
g_{j}\left(x_{j}^{\vartheta_{j}}, u_{j}^{v_{j}}\right) \leq 0
$$

The constraints referred to as the ships domains in marine navigation, may assume a shape of a circle, ellipse, hexagon, or parabola and may be generated for example - by an artificial neural network, see fig. 2 [1].
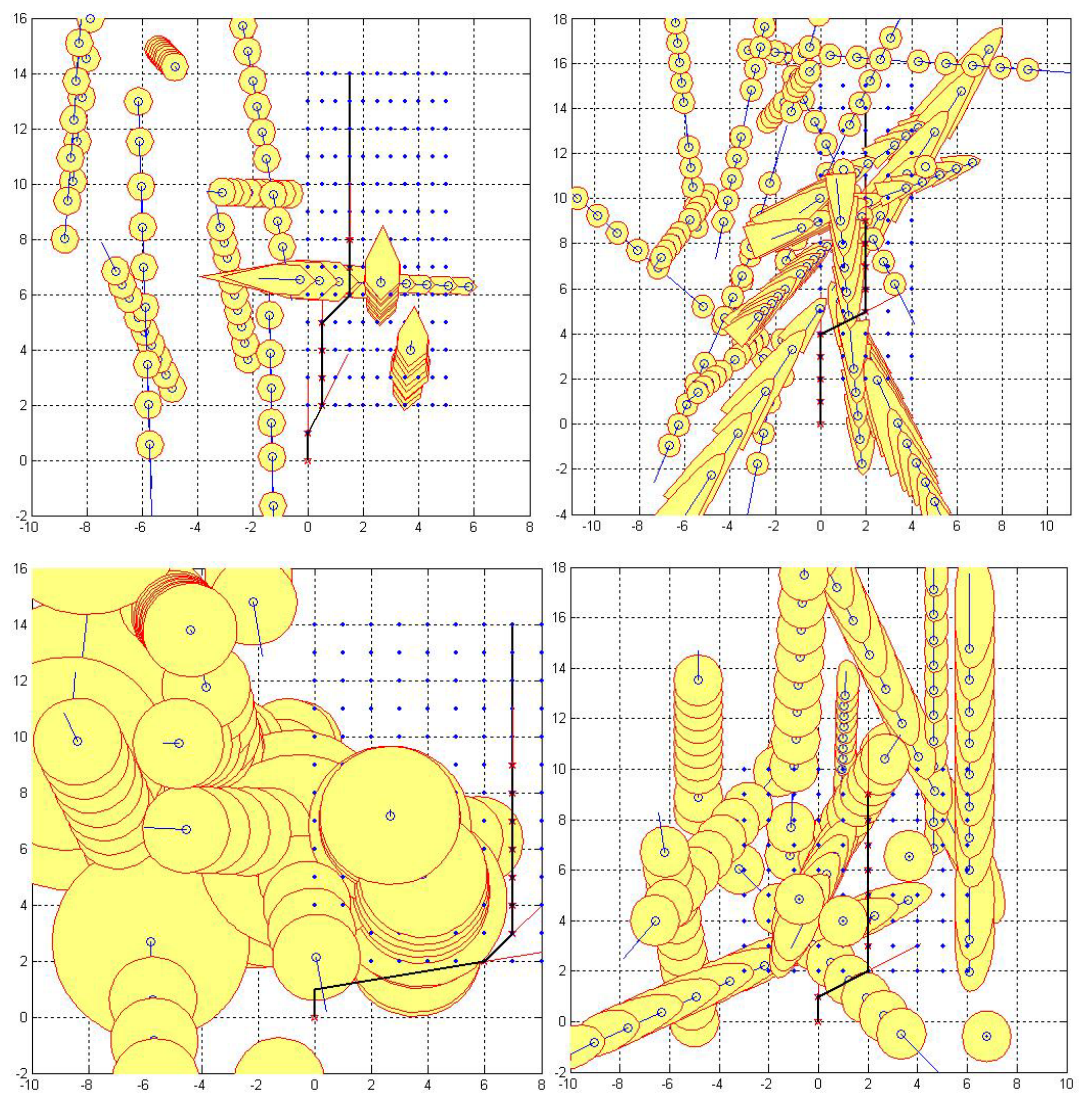

Figure 2: $\quad$ Forms of the neural ships domains.

\subsection{Quality index control}

The synthesis of the decision making pattern of the object control leads to the determination of the optimal strategies of the players who determine the most favourable, under given conditions, conduct of the process. For the class of non-coalition games, often used in the control techniques, the most beneficial conduct of the own control object as a player with $j$-th object is the minimization of her goal function in the form of the payments - the integral payment and the final one: 


$$
I_{0}^{j}=\int_{t_{0}}^{t_{k}}\left[x_{0}^{\vartheta_{0}}(t)\right]^{2} d t+r_{j}\left(t_{k}\right)+d\left(t_{k}\right) \rightarrow \min
$$

The integral payment represents loss of way by the ship while passing the encountered objects and the final payment determines the final risk of collision $r_{j}\left(t_{k}\right)$ relative to the $j$-th object and the final deflection of the ship $d\left(t_{k}\right)$ from the reference trajectory $[3,5,7]$.

\section{Control algorithms}

Each approximated model of the process may be assigned a respective method of safe control of a ship. The multi-stage positional game POSTRAJ and multi-step matrix game RISKTRAJ algorithms of safe ship control will be presented $[4,6]$.

\subsection{POSTRAJ algorithm}

The safe optimal control of the own ship $u_{0}^{*}(t)$, equivalent for the current position $p(t)$ to the optimal positional steering $u_{0}^{*}(p)$, is determined by:

- sets of acceptable strategies $U_{j}^{O}\left[p\left(t_{k}\right)\right]$ for the encountered $j$-th object relative to the own ship,

- a pair of vectors $u_{j}^{m}$ and $u_{0}^{j}$,

- the optimal positional strategy for the own ship $u_{0}^{*}(p)$ from the condition:

$$
I^{*}=\min _{u_{0} \in U_{0}=\bigcap_{j=I}^{m} U_{0}^{j}}\left\{\min _{u_{j}^{m} \in U_{j}} \min _{u_{0}^{j} \in U_{0}^{j}\left(u_{j}\right)} S_{0}\left[x_{0}\left(t_{k}\right), L_{k}\right]\right\}=S_{0}^{*}
$$

where $U_{0}$ - sets control of the own ship, $U_{j}-$ sets control of a met $j$-th object.

Function

$$
S_{0}\left[x_{0}(t), L_{k}\right]=\int_{t_{0}}^{t_{L_{k}}} u_{0}(t) d t
$$

is the own ship's control goal function which characterises the ship's distance at the moment $t_{0}$ to the closest point of turn $L_{k}$ on the assumed voyage route, see fig. 3.

The criterion for the selection of the optimal trajectory of the own ship is achieved by determining the ship's course and speed, which would ensure the smallest loss of way for a safe passing of the encountered objects, at a distance which is not smaller than the assumed value $D_{s}$, always with respect to the ship's dynamics in the form of the advance time to the manoeuvre $t_{m}$, with element $t_{m}^{\Delta \psi}$ during course manoeuvre $\Delta \psi$ or element $t_{m}^{\Delta V}$ during speed manoeuvre $\Delta V$. 


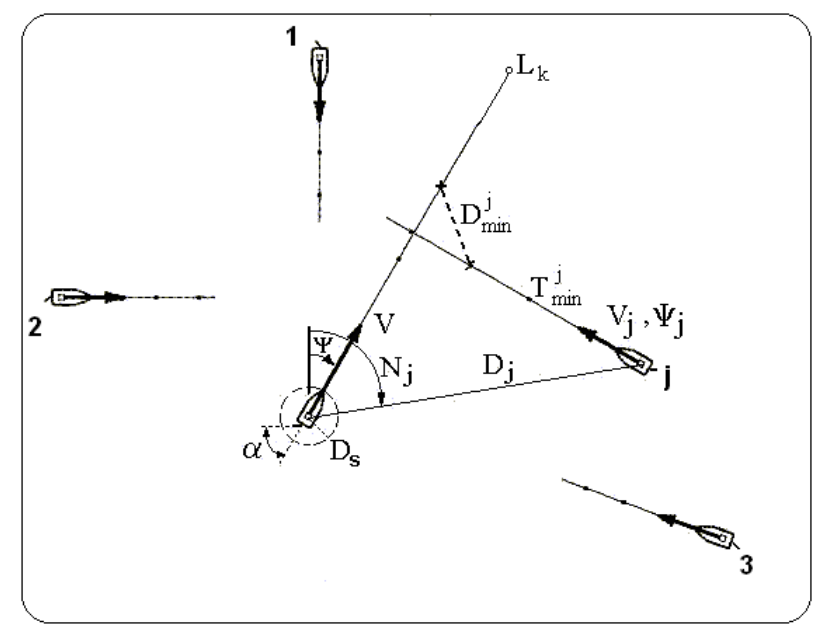

Figure 3: Navigational situation representing the passing of the own ship and $j$-th objects.

\subsection{RISKTRAJ algorithm}

The safe optimal control of the own ship $u_{0}^{*}(t)$ is the solution of a matrix game in which the own ship as player $\mathrm{O}$ has a possibility of using $s_{0}$ pure various strategies, and a $j$-th met object as player $\mathrm{J}$ has $s_{j}$ various pure strategies. Through the limitation of $s_{0}$ and $s_{j}$ strategies selection the COLREG requirements can be taken into consideration. In a dual problem player $\mathrm{O}$ aims to minimize the risk of collision $r_{j}$, while player $\mathrm{J}$ aims to maximize the risk. The value of the risk of the collision $r_{j}$ is defined as the reference of the actual approach situation at sea described by parameters $D_{\min }^{j}$ and $T_{\text {min }}^{j}$, to the assumed assessment of the situation defined as safe determined by safe distance $D_{s}$ and safe time $T_{s}$ of the approach:

$$
r_{j}=\frac{1}{\sqrt{w_{l}\left(\frac{D_{\text {min }}^{j}}{D_{s}}\right)^{2}+w_{2}\left(\frac{T_{\text {min }}^{j}}{T_{s}}\right)^{2}+w_{3}\left(\frac{D_{j}}{D_{s}}\right)^{2}}}
$$

where the weight coefficients $w_{1}, w_{2}$ and $w_{3}$ are dependent on the state of the visibility at sea, dynamic length, the dynamic beam of the ship and the kind of water region.

The components of the mixed strategy express the distribution of the probability of the players using their pure strategies. As a result, for the goal of control quality index in the form: 


$$
\left(I_{0}^{(j)}\right)^{*}=\min _{s_{0}} \max _{s_{j}} r_{j}
$$

probability matrix $\boldsymbol{P}\left[p_{j}\left(s_{j}, s_{0}\right)\right]$ of applying each one of the particular pure strategies is obtained. Applying the dual linear programming method, the solution of the safe optimal control problem is the strategy representing the highest probability:

$$
u_{0}^{*}(t)=u_{o}^{\left(v_{0}\right)}\left\{\left[p_{j}\left(s_{j}, s_{0}\right)\right]_{\max }\right\}
$$
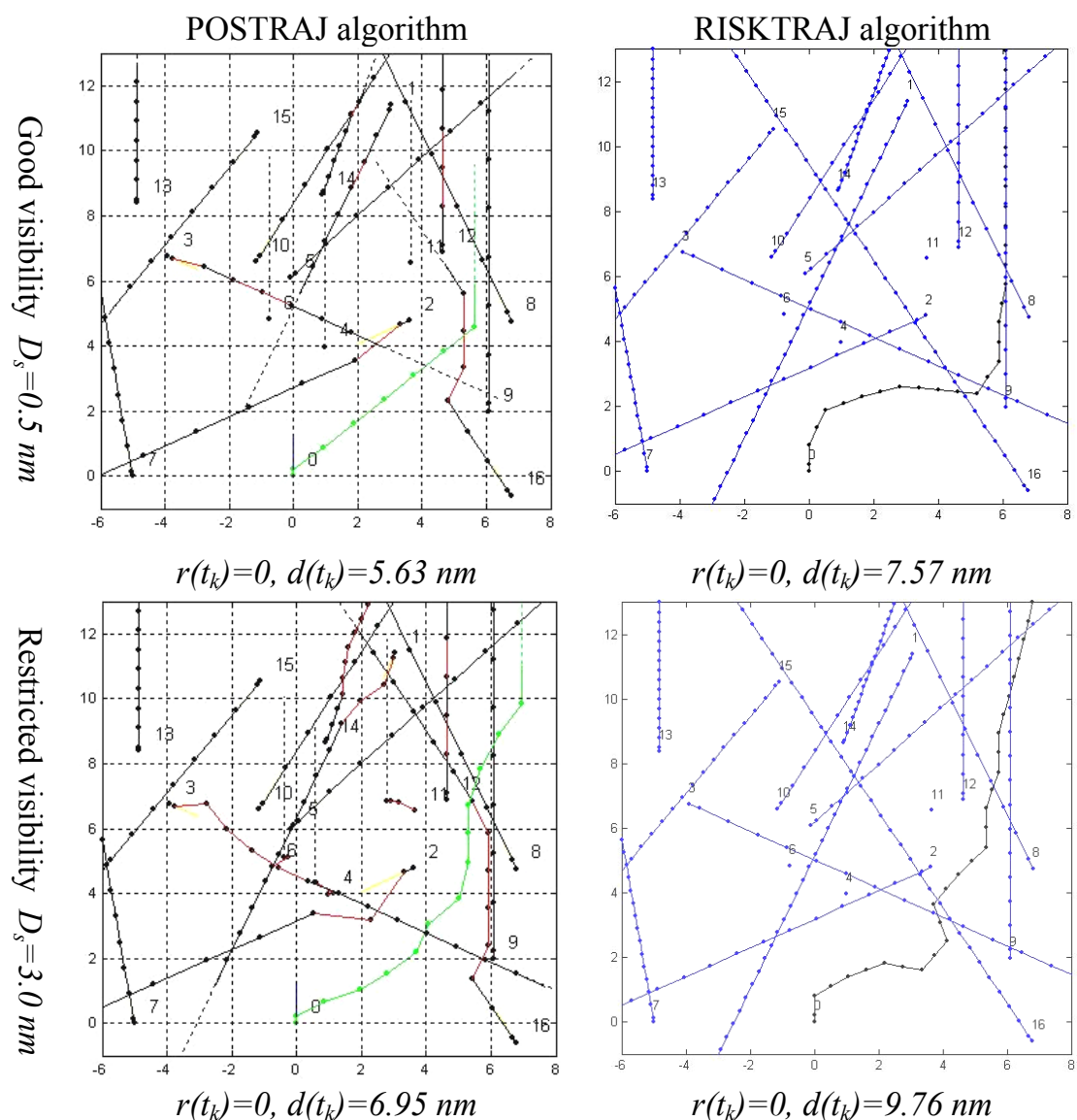

Figure 4: Positional and risk safe optimal game trajectories in situation on the Baltic Sea for $j=16$ encountered ships. 


\section{Computer simulation}

Computer simulation of positional trajectory POSTRAJ and risk trajectory RISKTRAJ control algorithms was carried out on in examples of real navigational situations of passing different numbers of encountered objects at sea. See figures 4-6.
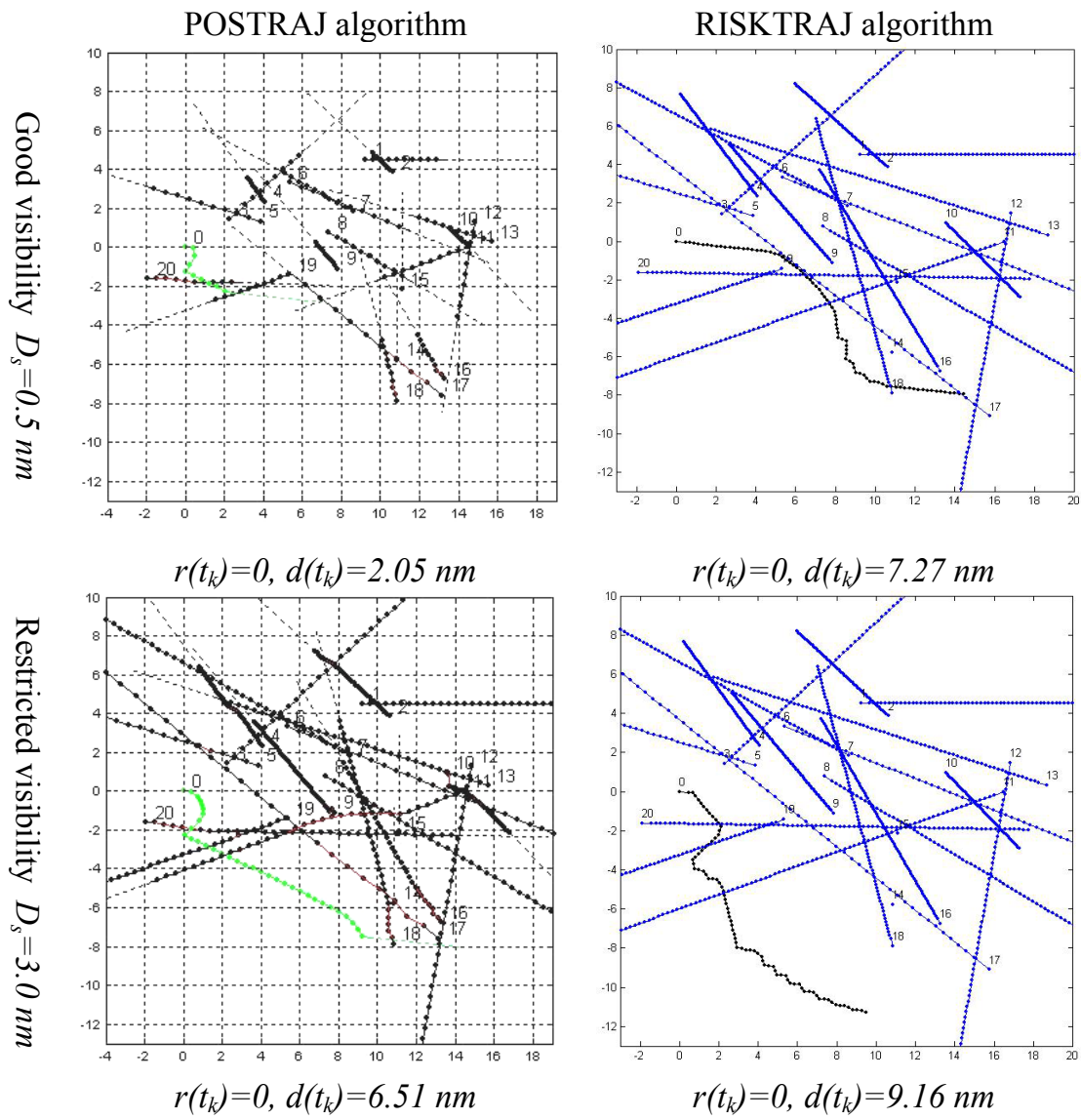

Figure 5: Positional and risk safe optimal game trajectories in situation on the North Sea for $j=20$ encountered ships.

\section{Conclusion}

POSTRAJ and RISKTRAJ control algorithms represent formal models of mental navigators leading the ship and making manoeuvring decisions. These algorithms can be used for the computer support of navigator safe manoeuvring decisions in collision situations using information from the ARPA anti-collision radar system. 

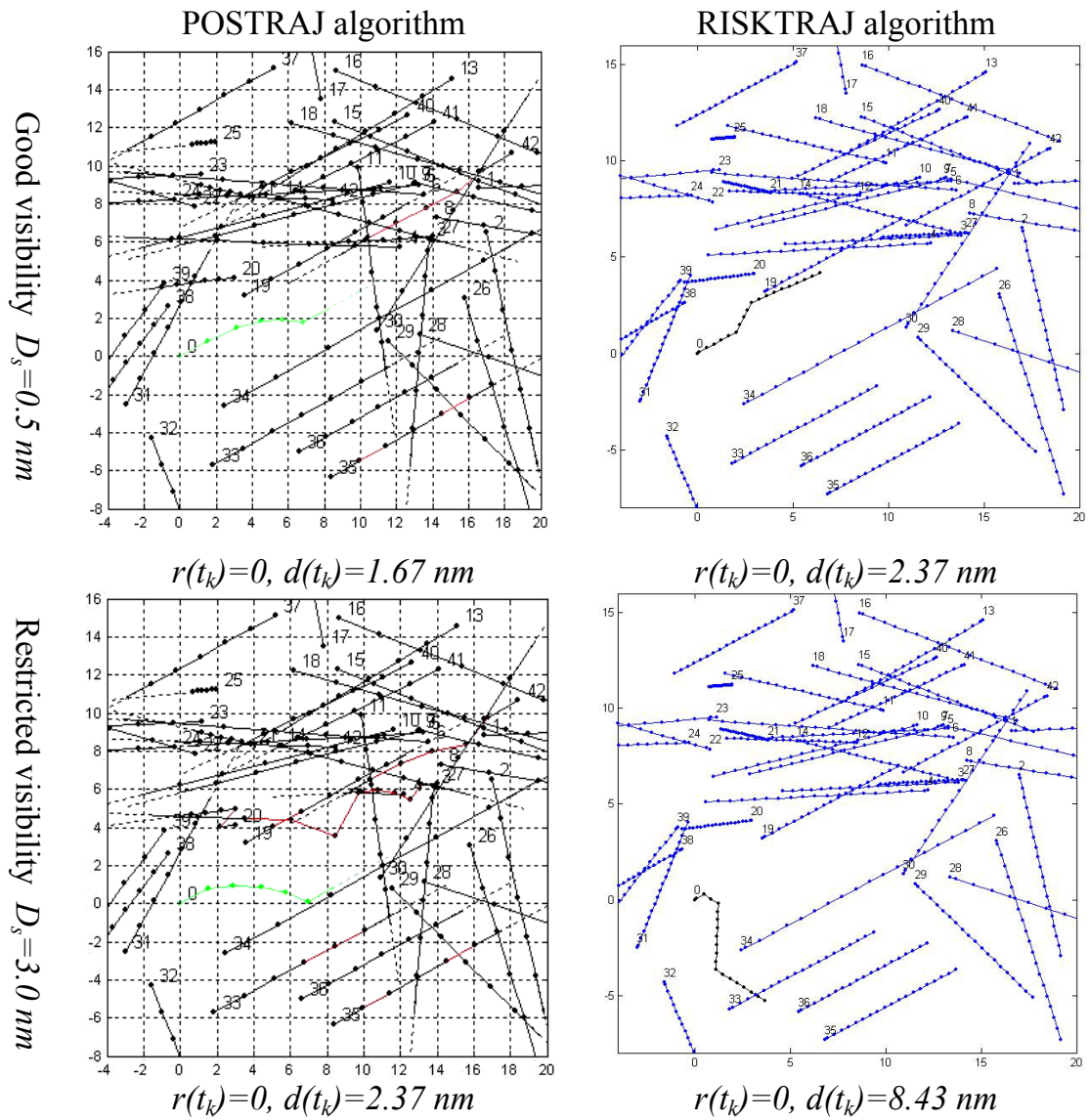

Figure 6: Positional and risk safe optimal game trajectories in situation in the English Channel for $j=42$ encountered ships.

\section{References}

[1] Baba, N. \& Jain, L.C., Computational Intelligence in Games, PhysicaVerlag: New York, 2001.

[2] Engwerda, J.C., LQ Dynamic Optimization and Differential Games, John Wiley and Sons: West Sussex, pp. 359-426, 2005.

[3] LaValle, S.M., Planning algorithms, Cambridge University Press: New York, pp. 357-586, 2006.

[4] Lisowski, J., Game control methods in navigator decision support system. Journal of Archives of Transport, 17, pp. 133-147, 2005.

[5] Lisowski, J., The dynamic game theory methods applied to ship control with minimum risk of collision, Risk Analysis VI, Computational Mechanics Publications: Southampton and Boston, pp. 293-302, 2006. 
[6] Lisowski, J., Application of dynamic game and neural network in safe ship control. Polish Journal of Environmental Studies, 16(4B), p. 114-120, 2007.

[7] Nisan, N., Roughgarden, T., Tardos, E. \& Vazirani, V.V., Algorithmic Game Theory, Cambridge University Press: New York, pp. 717-733, 2007.

[8] Osborne, M.J., An Introduction to Game Theory, Oxford University Press: New York and Oxford, pp. 313-356, 2004. 